\title{
Validation of the WINROP screening algorithm among preterm infants in East Malaysia
}

\author{
Nurhayati Abdul Kadir', Syed Shoeb Ahmad', Shuaibah Abdul \\ Ghani $^{1,2}$, Mae-Lyn Catherine Bastion ${ }^{3}$ \\ 'Ophthalmology Department, Queen Elizabeth Hospital, Kota Kinabalu, \\ Malaysia; ${ }^{2}$ Pediatric Ophthalmology Unit, Sabah Women and Children's Hospital, \\ Kota Kinabalu, Malaysia; ${ }^{3}$ Department of Ophthalmology, Universiti Kebangsaan \\ Malaysia, Selangor, Malaysia
}

\begin{abstract}
Objective: To prospectively validate the WINROP (Weight, Insulin-like growth factor 1, Neonatal, Retinopathy of Prematurity) screening algorithm (www.winrop.com) based on longitudinal measurements of neonatal body weights in predicting the development of severe retinopathy of prematurity (ROP) among preterm infants admitted to the neonatal intensive care unit of a tertiary care center in East Malaysia.

Methods: All premature infants of less than 32 weeks gestational age (GA) were included in this cohort. Their body weight was measured weekly from birth to 36 weeks postmenstrual age and entered into the computer-based surveillance system: WINROP. Infants were then classified by the system into high-or low-risk alarm group. The retinopathy findings were recorded according to Early Treatment for ROP criteria. However, the screening and management of infants were done according to the recommendations of the Continuous Practice Guidelines, Ministry of Health, Malaysia. The team members involved in screening and those recording the findings were kept blinded from each other.

Results: A total of 151 infants with median GA at birth of 30 weeks (interquartile range $[I Q R] \pm 2.1)$ and mean birth weight of 1,264 $\mathrm{g}$ (standard deviation \pm 271 ) were analyzed. High-risk alarm was signaled in 85 (56.3\%) infants and 9 (6.6\%) infants developed type 1 $R O P$. One infant in the low-risk alarm group developed type 1 ROP requiring laser retinal photocoagulation. The median time lag from the high-risk alarm signal to the development of type 1 ROP was 10.4 (IQR \pm 8.4 ) weeks.

Conclusion: In this cohort, the WINROP algorithm had a sensitivity of $90 \%$, with negative predictive value of $98.5 \%$ (95\% confidence interval) for detecting infants with type 1 ROP and was able to predict infants with ROP earlier than their due screening date. This study shows that a modified version of the WINROP algorithm aimed at specific populations may improve the outcome of this technique.
\end{abstract}

Keywords: infant, insulin-like growth factor 1, premature, retina, retinopathy of prematurity $(R O P)$

Correspondence: Syed Shoeb Ahmad, Department of Surgery, Ajmal Khan Tibbiya

College, Aligarh, India.

Email: syedshoebahmad@yahoo.com 


\section{Introduction}

Retinopathy of prematurity (ROP) remains a common cause for pediatric visual impairment and blindness worldwide. The Cryotherapy for ROP study reported that $65.8 \%$ of infants with birth weight less than $1,251 \mathrm{~g}$ developed ROP.' In a similar group, the Early Treatment for ROP (ETROP) study had given an incidence of ROP at $68 \% .{ }^{2}$ In a study from Malaysia, Choo et al. reported the incidence of ROP in this country to be $58.6 \%$ in extremely low birth weight infants. ${ }^{3}$ In the ETROP study, $8 \%$ of infants with ROP required treatment. ${ }^{2}$ ROP remains an important cause for preventable blindness in children. A study by Rahi and Cable reported ROP to be responsible for almost $3 \%$ of all childhood blindness. ${ }^{4}$ This number could be higher in developing countries. These data confirm the existence of a huge burden of ROP in vulnerable populations and the need to develop better screening strategies to manage this condition.

Traditionally, screening recommendations for ROP have emphasized strict follow-up visits to diagnose the condition. However, these protocols suffer from some shortcomings, including the stressful nature of the examination for infants, carers, and practitioners; the economic burden on health services; maintaining standby neonatal intensive care unit (NICU) services, with the need for experts to identify and treat the condition if required. ${ }^{5,6} \mathrm{It}$ is imperative to find better screening techniques and protocols to overcome these deficiencies, especially in underserved areas. One such online-based surveillance system that has gained attention is the WINROP. The acronym WINROP (Weight, Insulin-like growth factor 1 , Neonate, ROP) indicates the correlation between early postnatal weight gain and insulin-like growth factor 1 (IGF-1) in the development of severe ROP. A number of studies performed, mainly in developed countries, have shown the high sensitivity and specificity of this system in raising alarms, which direct the practitioner to a need for intervention. ${ }^{7,8}$ In a further simplification of the WINROP system, only the weight of the preterm infant is monitored. ${ }^{9-11}$ This refinement would further facilitate the WINROP method. However, this modification needs to be validated extensively and widely across different population groups in order to confirm its effectiveness. ${ }^{12-14}$

We conducted a longitudinal cohort study on 151 preterm infants admitted to the NICU of a tertiary care facility in East Malaysia. This study assessed the modified WINROP method in monitoring the postnatal weight gain and capturing the data into the online surveillance system. Alarms were raised when any intervention was required. Our results show that this modified WINROP algorithm has a sensitivity of $90 \%$ with negative predictive value of $98.5 \%$ (95\% confidence interval $[\mathrm{Cl}]$ ) in detecting infants with type 1 ROP. Thus, this modality can be an effective means of screening infants for ROP. 


\section{Methods}

\section{Patients}

A prospective cohort study was performed between May 2015 and March 2016 on premature infants born at a gestational age (GA) of less than 32 weeks, seen at a tertiary care hospital in East Malaysia. All infants in the study were admitted to the NICU, written informed consent for inclusion in the study was obtained from the parents, daily weight was measured, and the infants screened for ROP. Premature infants who had incomplete weight measurement until 36 weeks postmenstrual age, nonphysiological weight gain, or retinal disease other than ROP were excluded from this study.

The GA of participating infants was determined by obstetricians and neonatologists based on last menstrual date of the infant's mother, early antenatal ultrasonography, and/or neonatal physical assessment using the New Ballard Score. ${ }^{15}$ The body weight of premature infants was measured from the day of birth until discharge from hospital using a validated infant weighing scale. The data collected included GA at birth, body weight, weekly postnatal weight measurements conducted until 36 weeks postmenstrual age, sex, parity, race, ROP examination results, and incidence of bronchopulmonary dysplasia (BPD), intraventricular hemorrhage, necrotizing enterocolitis, or neonatal sepsis.

\section{ROP screening and treatment}

All the participating infants were examined according to the Clinical Practice Guidelines by the Ministry of Health Malaysia for ROP screening. ${ }^{16}$ The first ROP examination was performed between fourth and sixth weeks postnatal age, or for very premature infants, at 31 to 33 weeks of GA and repeated every 2 weeks or more according to the severity of ROP. The examination was continued until full vascularization of the peripheral retina, regression of ROP, occurrence of type 1 ROP, or the need for treatment was achieved. The ROP examinations were performed by a qualified pediatric ophthalmologist using the standard binocular indirect ophthalmoscope with a $20 \mathrm{D}$ or $28 \mathrm{D}$ condensing lens. Prior to the examination, the pupil was dilated using cyclopentolate $0.5 \%$ and phenylephrine $2.5 \%$ eye drops.

The ROP findings were classified according to the International Classification of Retinopathy of Prematurity, revisited 2005 (stages 1-5). ${ }^{17}$ The highest stage of ROP, the lowest zone, presence of prethreshold or threshold disease, and the need for treatment were recorded. Prethreshold ROP was further subclassified according to the ETROP criteria into type 1 or type 2 ROP. ${ }^{2}$

Type 1 ROP is defined as any zone I ROP with plus disease, zone I stage 3 ROP without plus disease, or zone II stage 2 or 3 ROP with plus disease. Type 2 ROP is defined as zone I stage 1 or 2 ROP without plus disease or zone II stage 3 ROP without plus disease. If the infant developed type 1 ROP during the study, 
standard treatment with laser photocoagulation was given.

\section{WINROP screening}

The WINROP algorithm was developed using the method of online statistical surveillance system (www.winrop.com) that permits the user to enter body weight, GA, date of birth, and successive weight measurements; allows tracking of multiple infants; and provides a user-friendly indication of low- or high-risk status for developing sight-threatening ROP. The requirements for using the WINROP algorithm system are:

1. GA less than 32 weeks at birth;

2. weekly weight measurement;

3. physiological weight gain;

4. absence of other pathological retinal vascular disease.

The WINROP algorithm is based on expected weight gain data obtained from premature infants with no ROP or stage 1 ROP, and then calculating the difference from the observed values. ${ }^{7}$ The differences or deviations between the expected weight gain and the actual weight gain are accumulated weekly. When these cumulative deviations surpass a threshold signal level, an alarm is signaled, indicating that the premature infant is at risk for developing severe ROP. Thus, the WINROP outcome is either no alarm or alarm.

In our study, the weekly body weight of participating premature infants was measured by an observer blinded from the ROP and WINROP outcome, then entered into the WINROP online website by another person who was also unaware of the ROP outcome. The WINROP algorithm system calculates the risk weekly to predict infants who are at risk of developing severe ROP. Based on the WINROP analysis, the participating infants were categorized into two groups: no (also called low-risk) alarm group, who are less likely to develop type 1 ROP, and high-risk alarm group, who are at risk of developing type 1 ROP. The screening and management of the infants were done according to the Continuous Practice Guidelines formulated by the Ministry of Health, Malaysia. The alarm status was recorded by the main author; however, the screening protocol was kept unchanged and followed the guidelines. As this was a double blinded study, the team members involved in screening and the persons involved in data entry or alarm acquisition remained unaware of the outcomes of each other. Ethical approval for this study was obtained from the ethics committee of University Kebangsaan Malaysia.

\section{Statistical analysis}

The sensitivity and specificity of WINROP screening to identify premature infants who developed type 1 ROP were analyzed with Statistical Package for the Social Sciences. The negative and positive predicted values were calculated using the 
sensitivity, specificity, and prevalence of type 1 ROP for the participating premature infants. We calculated $95 \% \mathrm{Cl}$ for estimated binary proportions (sensitivity and specificity).

\section{Results}

\section{Patients}

A total of 151 infants admitted in the NICU of a tertiary care hospital participated in this study and were screened for ROP. In this cohort, male and female

Table 1. Patient demographic data

\begin{tabular}{|l|l|}
\hline Birth characteristics & No. (\%) \\
\hline GA, median (IQR), weeks & $30( \pm 2.1)^{\mathrm{a}}$ \\
\hline BW, mean (SD), g & $1,264( \pm 271)$ \\
\hline Sex & $77(51)$ \\
\hline Male & $74(49)$ \\
\hline Female & \\
\hline Birth multiplicity & $130(86)$ \\
\hline Single & $21(14)$ \\
\hline Twin & \\
\hline
\end{tabular}

BW: birth weight

askewed to the left

Table 2. Prematurity-associated comorbidities

\begin{tabular}{|l|l|l|l|l|}
\hline \multirow{2}{*}{ Characteristic } & \multicolumn{4}{l}{ Infants, no. } \\
\cline { 2 - 6 } & No ROP & Non-PT ROP & Type 1 ROP & Total infants \\
\hline BPD & 83 & 9 & 10 & 102 \\
\hline $\begin{array}{l}\text { Intraventricular } \\
\text { hemorrhage }\end{array}$ & 20 & 6 & 4 & 30 \\
\hline Necrotizing enterocolitis & 4 & 0 & 0 & 4 \\
\hline Neonatal sepsis & 80 & 9 & 5 & 94 \\
\hline
\end{tabular}

PT: prethreshold 
patients were almost equal in number, with median GA at birth of 30 weeks (interquartile range $[\mathrm{IQR}] \pm 2.1$ ) and mean body weight of 1,264 $\mathrm{g}$ (standard deviation $[S D] \pm 271$ ). Singleton births were recorded in $86 \%$ infants and the remaining were twins. The birth characteristics of the study population are shown in Table 1.

There was a statistically significant association between BPD and type 1 ROP $(p=0.031)$ as all infants with type 1 ROP were diagnosed with BPD. There were no significant differences in intraventricular hemorrhage, necrotizing enterocolitis, and neonatal sepsis with the severity of ROP (Table 2 ).

\section{ROP and WINROP outcome}

Type 1 ROP developed in 10 infants (6.6\%), with a median GA at birth of 27.8 weeks $(\mathrm{IQR} \pm 2.7)$ and mean body weight of $1,047 \mathrm{~g}(\mathrm{SD} \pm 272)$. The diagnosis of type 1 ROP was made at median GA of 39.7 weeks (range, 37.6-44.6 weeks). Infants in this group were treated with laser retinal photocoagulation within 72 hours of the diagnosis. Three of these required repeated laser treatments and none of them progressed to retinal detachment. Non-prethreshold ROP developed in 12 infants (7.9\%), with median GA at birth being 27.7 weeks (IQR \pm 1.9 ) and mean body weight of $1,029 \mathrm{~g}(S D \pm 137)$. Most of the infants in the non-prethreshold group were in stage 1 and 2 at zone II ROP, which regressed spontaneously without treatment. No ROP occurred in $85 \%$ of the premature infants. The median GA at birth was 30.1 weeks (IQR \pm 1.8$)$ and mean body weight was $1,303 \mathrm{~g}(\mathrm{SD} \pm 263)$ (Table 3).

Table 3. Alarm signal in relation to ROP categories and birth characteristics

\begin{tabular}{|c|c|c|c|c|}
\hline & \multicolumn{2}{|l|}{ Alarm status } & \multirow{2}{*}{ All infants } \\
\hline & & Low risk & High risk & \\
\hline \multicolumn{2}{|l|}{ Infants, no. (\%) } & $66(43.7)$ & $85(56.3)$ & 151 \\
\hline \multirow{3}{*}{$\begin{array}{l}\text { ROP categories, } \\
\text { no. }\end{array}$} & No ROP & 64 & 65 & 129 \\
\hline & Non-PT ROP & 1 & 11 & 12 \\
\hline & Type 1 ROP & 1 & 9 & 10 \\
\hline \multirow{2}{*}{$\begin{array}{l}\text { Birth } \\
\text { characteristics }\end{array}$} & $\begin{array}{l}\text { GA, median (IQR) } \\
\text { weeks }\end{array}$ & $31.0( \pm 1.1)^{\mathrm{a}}$ & $29.1( \pm 2.0)^{\mathrm{a}}$ & $30( \pm 2.1)^{\mathrm{a}}$ \\
\hline & BW, mean $(S D) g$ & $1,518( \pm 157)$ & $1,067( \pm 152)$ & $1,264( \pm 271)$ \\
\hline
\end{tabular}

BW: birth weight; PT: prethreshold

askewed to the left 
Validation of WINROP screening algorithm among preterm infants in East Malaysia

Table 4. Sensitivity, specificity, PPV, NPV in predicting type 1 ROP using the WINROP algorithm

\begin{tabular}{|l|l|l|l|}
\hline \multirow{2}{*}{ Alarm status } & \multicolumn{2}{|l|}{ ROP categories, no. } & \multirow{2}{*}{ Predictive values } \\
\cline { 2 - 4 } & $\begin{array}{l}\text { Type 1 } \\
(\mathbf{n}=\mathbf{1 0})\end{array}$ & $\begin{array}{l}\text { Non-type 1 } \\
(\mathbf{n}=\mathbf{1 4 1})\end{array}$ & $\begin{array}{l}\text { PPV }=10.6 \%, \\
95 \% \mathrm{Cl}: 6,19\end{array}$ \\
\hline $\begin{array}{l}\text { High risk } \\
(\mathrm{n}=85)\end{array}$ & 9 & 76 & $\begin{array}{l}\mathrm{NPV}=98.5 \%, \\
95 \% \mathrm{Cl}: 92,99\end{array}$ \\
\hline $\begin{array}{l}\text { Low risk } \\
(\mathrm{n}=66)\end{array}$ & 1 & 65 & \\
\hline & $\begin{array}{l}\text { Sensitivity 90\%, } \\
95 \% \text { Cl: 60,98 }\end{array}$ & $\begin{array}{l}\text { Specificity 46\%, } \\
95 \% \mathrm{Cl}: 38,54^{\mathrm{a}}\end{array}$ \\
\hline
\end{tabular}

NPV: negative predictive value; PPV: positive predictive value

A high-risk alarm was signaled in $56.3 \%$ of infants, of which 9 infants developed type 1 ROP and 11 infants developed non-prethreshold ROP. Type 1 ROP was treated with laser retinal photocoagulation, while non-prethreshold ROP regressed and resolved spontaneously. Most of the type 1 ROP infants were diagnosed before 40 weeks of GA, except two infants who developed type 1 ROP after 45 weeks of GA and were treated. A low-risk alarm developed mostly in no-ROP premature infants. One infant in the low-risk alarm group who developed type 1 ROP was born at 30.6 weeks GA and birth weight was $1,700 \mathrm{~g}$ with severe $B P D$, requiring prolonged intubation for more than 3 weeks. ROP was identified at 36 weeks of GA and treated with laser photocoagulation. Another infant in the non-prethreshold group had a high-risk alarm and developed stage 1 ROP, which then regressed spontaneously. The median GA at birth and body weight were lower in the high-risk alarm group than in the low-risk alarm group (Table 3).

The median time from birth to high-risk alarm signaled was 2 weeks (IQR \pm 2.0 ) and from high-risk alarm signaled to development of type 1 ROP was 10.4 weeks $(\mathrm{IQR} \pm 8.4)$.

\section{Test characteristics}

In this prospective cohort, the sensitivity of the WINROP algorithm in predicting severe ROP was $90 \%$ ( $95 \% \mathrm{Cl}, 60 \%-98 \% ; 9$ of 10 infants) and the specificity was $46 \%$ (95\% Cl, 38\%-54\%; 65 of 141 infants). The positive predictive and negative predictive values in this study group were $10.6 \%$ and $98.5 \%$, respectively (Table 4 ).

\section{Discussion}

Worldwide, there is an uptrend in the number of infants being diagnosed with ROP, especially so in the developed and higher strata developing areas. ${ }^{18,19}$ 
This has been directly attributed to better survival rates of premature infants as a consequence of advances in neonatal care and better access to NICUs. In underdeveloped areas, poor neonatal services are responsible for higher infant mortality rates among preterm babies, which may lead to lower overall ROP rates. In Malaysia, the Ministry of Health has set out specific guidelines regarding screening of premature infants. ${ }^{16}$ All babies who are less than 32 weeks GA or with a birth weight of less than $1,500 \mathrm{~g}$ or infants with an unstable clinical course who are at high risk (as determined by the neonatologist or pediatrician) need to be screened until the retina is fully vascularized, or there is regression of ROP or need for treatment. In our study, on average, an infant was subjected to the screening procedure at least 5 to 6 times if there was no ROP and more frequently in cases where ROP was detected. This produces severe stress on the fragile preterm infants, as well as on the healthcare system. ${ }^{20} \mathrm{~A}$ study by Laws et al. has documented a rise in systolic and diastolic blood pressure as well as a fall in oxygen saturation in infants during ROP screening. ${ }^{5}$

Early diagnosis and management of ROP by lasers and anti-vascular endothelial growth factor agents has reduced the incidence of blindness by $25 \%$ in infants diagnosed with late-stage ROP. However, these treated infants continue to have poor visual acuity following treatment, while ocular and visual development is significantly impaired. ${ }^{21}$ About $70 \%$ of infants with ROP develop myopia during the first year after birth. ${ }^{22}$ Astigmatic refractive errors are seen in $40 \%$ of eyes having a history of ROP, while strabismus affects $20 \%$ infants diagnosed with ROP. ${ }^{21}$ These pathologies need to be addressed by an earlier diagnosis of ROP.

The WINROP algorithm is an ROP screening protocol based on weekly postnatal weight measurements and assessment of IGF-1 levels to predict the development of severe ROP in premature infants. ${ }^{7}$ The system uses a cumulative deviation statistical approach. Every week the infant's actual weight is compared to an expected growth curve of infants who developed no or mild ROP. The differences or deviations between the expected weight and the actual weight are accumulated from week to week. When these cumulative deviations surpass a threshold, an alarm is signaled, indicating the preterm infant is at risk for developing sight-threatening complications of ROP. In a further modification of the procedure, only the weight gains are taken into consideration. ${ }^{8}$ By avoiding the weekly blood sampling to assess IGF-1, this modification makes the procedure simpler, safer, and more economical.

The sensitivity of the WINROP system has shown variations among countries and populations, being high in developed countries and slightly lower in developing countries and Asian populations. ${ }^{8-14}$ In this study, the modified WINROP algorithm correctly identified 9 of 10 (90\%) infants who had developed type 1 ROP that required treatment. The sensitivity of the WINROP algorithm was found to be as high as in the Korean study $(90 \%)^{13}$ and higher than the Chinese study $(87.5 \%)^{14}$ 
when evaluated in Asian countries. Every country has different demographics and differences in terms of birth weight and postnatal weight gain according to their GA. Asian populations (Malaysia) are small and light in body weight as compared to European populations. Thus, in this study the sensitivity was high/comparable to other studies; however, the specificity is much lower. In this cohort, among infants who developed type 1 ROP, the high-risk alarm was signaled at a median of 2 weeks after birth and 10 weeks before the diagnosis of type 1 ROP. The high sensitivity and early identification of infants at high risk of developing type 1 ROP before the scheduled screening showed that it can be used as an adjunct for conventional ROP screening protocols.

\section{Conclusion}

Our study validates the successful implementation of the modified WINROP algorithm in a population of preterm infants seen in East Malaysia. This protocol can prove to be a useful tool to modify the current ROP screening guidelines, wherein an infant with no alarm can be examined less often, while an infant with a high-risk alarm signal can be screened as per guidelines. This would save these fragile infants from unnecessary examinations and direct the efforts of the staff toward the sicker infants who require more attention.

\section{References}

1. Cryotherapy for Retinopathy of Prematurity Cooperative Group. Multicenter trial of cryotherapy for retinopathy of prematurity: ophthalmological outcomes at 10 years. Arch Ophthalmol. 2001;119:1110-1118.

2. Early Treatment for Retinopathy of Prematurity Cooperative Group. Revised indications for the treatment of retinopathy of prematurity: results of the early treatment for retinopathy of prematurity randomized trial. Arch Ophthalmol. 2003;121:1684-1694.

3. Choo MM, Martin FJ, Theam LC, et al. Retinopathy of prematurity in extremely low birth weight infants in Malaysia. J AAPOS. 2009;13:446-449.

4. Rahi JS, Cable N. Severe visual impairment and blindness in children in the UK. Lancet. 2003;362:1359-1365.

5. Laws DE, Morton C, Weindling M, et al. Systemic effects of screening for retinopathy of prematurity. Br J Ophthalmol. 1996;80:425-428.

6. Mitchell AJ, Green A, Jeffs DA, et al. Physiologic effects of retinopathy of prematurity screening examinations. Adv Neonatal Care. 2011;11:291-297.

7. Löfqvist C, Andersson E, Sigurdsson J, et al. Longitudinal postnatal weight and insulin-like growth factor I measurements in the prediction of retinopathy of prematurity. Arch Ophthalmol. 2006;124:1711-1718.

8. Löfqvist C, Hansen-Pupp I, Andersson E, et al. Validation of a new retinopathy of prematurity screening method monitoring longitudinal postnatal weight and insulinlike growth factor I. Arch Ophthalmol. 2009;127:622-627.

9. Hellstrom A, Hard AL, Engstrom E, et al. Early weight gain predicts retinopathy in preterm infants: new, simple, efficient approach to screening. Pediatrics. 2009;123:e638-e645. 
10. Wu C, Vanderveen DK, Hellström A, et al. Longitudinal postnatal weight measurements for the prediction of retinopathy of prematurity. Arch Ophthalmol. 2010;128:443-447.

11. Wu C, Löfqvist C, Smith LE, et al; WINROP Consortium. Importance of early postnatal weight gain for normal retinal angiogenesis in very preterm infants: a multicenter study analyzing weight velocity deviations for the prediction of retinopathy of prematurity. Arch Ophthalmol. 2012;130:992-999.

12. Hård A, Löfqvist C, Fortes Filho JB, et al. Predicting proliferative retinopathy in a Brazilian population of preterm infants with the screening algorithm WINROP. Arch Ophthalmol. 2010;128:1432-1436.

13. Choi J, Löfqvist C, Hellström A, et al. Efficacy of the screening algorithm WINROP in a Korean population of preterm infants. JAMA Ophthalmol. 2013;131:62-66.

14. Sun $\mathrm{H}$, Kang W, Cheng $X$, et al. The use of the WINROP screening algorithm for the prediction of retinopathy of prematurity in a Chinese population. Neonatology. 2013;104:127-132.

15. Ballard JL, Khoury JC, Wedig K. New Ballard Score, expanded to include extremely premature infants. J Pediatr. 1991;119:417-423.

16. Malaysia Clinical Practice Guidelines for Retinopathy of Prematurity 2005. Available from: http:// www.acadmed.org.my/index.cfm?\&menuid $=67$

17. Classification of Retinopathy of Prematurity. The international classification of retinopathy of prematurity revisited. Arch Ophthalmol. 2005;123:991-999.

18. Hack M, Fanaroff AA. Outcomes of children of extremely low birthweight and gestational age in the 1990s. Semin Neonatol. 2000;5:89-106.

19. Blenow M, Ewald U, Fritz T, et al. One-year survival of extremely preterm infants after active perinatal care in Sweden. J Am Med Assoc. 2009;301:2225-2233.

20. Slevin M, Murphy J, Daly L, et al. Retinopathy of prematurity screening, stress related responses, the role of nesting. Br J Ophthalmol. 1997;81:762-764.

21. Chen J, Stahl A, Hellstrom A, et al. Current update on retinopathy of prematurity: screening and treatment. Curr Opin Pediatr. 2011;23:173-178.

22. Salvin JH, Lehman SS, Jin J, et al. Update on retinopathy of prematurity: treatment options and outcomes. Curr Opin Ophthalmol. 2010;21:329-334. 